\title{
Constrained Portfolio Optimisation: the state-of-the-art Markowitz Models
}

\author{
Yan Jin, Rong Qu and Jason Atkin \\ ASAP Group, School of Computer Science, The University of Nottingham, Nottingham, UK \\ $\{y w j, r x q, j a a\} @ c s . n o t t . a c . u k$
}

\begin{abstract}
Keywords: Constrained Portfolio Optimisation, Mean-Variance, Cardinality, Pre-assignment, Round-lot, Class
Abstract: $\quad$ This paper studies the state-of-art constrained portfolio optimisation models, using exact solver to identify the optimal solutions or lower bound for the benchmark instances at the OR-library with extended constraints. The effects of pre-assignment, round-lot, and class constraints based on the quantity and cardinality constrained Markowitz model are firstly investigated to gain insights of increased problem difficulty, followed by the analysis of various constraint settings including those mostly studied in the literature. The study aims to provide useful guidance for future investigations in computational algorithms.
\end{abstract}

\section{INTRODUCTION}

Portfolio optimisation (PO) is an extensively studied area in finance. The seminal work by (Markowitz, 1952) had a profound impact on the development of PO in the last 60 years. The Mean-Variance (MV) model introduced by Markowitz focusses on finding the best trade-off between the return and risk of portfolios, i.e. mean of return and covariance of return, to minimise the risk given an expected return level or vice versa.

Although the significance of the MV model is unanimously recognised, the basic model has been widely challenged for some underlying assumptions. It neglects many realistic restrictions faced by investors like tax and transaction cost; personal or strategic investment decisions, etc. It assumes assets are traded at any fractions. It implicitly encourages holdings of as many assets as possible to diversify the overall risk. In reality, an investment manager may face the restrictions on the minimum and/or maximum capital allocated to an asset or industry. Investors also prefer a limited number of assets (Jansen and van Dijk, 2002).

The complexity of the PO problem to a large extent depends on the constraints (Maringer, 2008). The basic MV model is a standard quadratic programming problem. There has been numerous tools to solve it optimally. However real-world financial constraints significantly increase the level of complexity. For instance, cardinality constraint requires only a limited number of assets to be included in the portfolio, which turns the problem into non-convex. It is no longer always suitable to use exact methods to find optimal solutions thus the majority of work in the current literature has focused on heuristics for the constrained PO problem.

Nevertheless, due to the fast development some constraints now can be handled by commercial solvers such as CPLEX with limited computational cost for many difficult optimisation problems. The purpose of our study is to provide an insight into the current state of the MV models with subsets of practical constraints using CPLEX, and provide useful guidance of potential areas of future research on computational algorithms for constrained PO problems.

This paper is organised as follows. Firstly, we overview the basic MV model and the extended constraints in Section 2. Then a comprehensive overview of related literature for various settings of practical constraints is presented in Section 3. It summarises the representative works in terms of different constraints applied. Section 4 presents the experimental study by reviewing the models in the literature and provides the optimal solutions or lower bound for those mostly studied models. Finally, in Section 5, we identify some possible future research on algorithms for the constrained MV models in portfolio optimisation. 


\section{PROBLEM FORMULATION}

\subsection{Mean-Variance Model}

The MV model considers a single period of investment. The process of PO allocates among $N$ different assets the proportions $\left(x_{i}, i=1, \ldots N\right)$ of the capital to form a portfolio. Each asset $i$ has a return rate $r_{i}$ and is associated with a covariance $\sigma_{i j}$ of the return with each other asset $j$. The total return $r_{P}$ of a portfolio is given by the weighted combination of the constituent assets' returns $\sum_{i=1}^{N} x_{i} r_{i}$, and its risk $v_{P}$ is defined by $\sum_{i=1}^{N} \sum_{j=1}^{N} \sigma_{i j} x_{i} x_{j}$. The aim is to minimize the portfolio risk $v_{P}$ for a given level of expected return $R_{\text {exp }}$ or vice versa, mathematical formulation of the model as follows:

\section{Minimise}

$$
v_{P}=\sum_{i=1}^{N} \sum_{j=1}^{N} \sigma_{i j} x_{i} x_{j}
$$

Subject to

$$
\begin{aligned}
& r_{P}=\sum_{i=1}^{N} x_{i} r_{i}=R_{\exp } \\
& \sum_{i=1}^{N} x_{i}=1 \\
& 0 \leqslant x_{i} \leqslant 1, i=1, \ldots, N
\end{aligned}
$$

Constraint (2) defines the expected return. The budget constraint (3) requires the whole capital should be invested. Investment to each asset is nonnegative, defined in constraint (4). A set of optimal portfolios of the lowest risk for various values of $R_{\text {exp }}$ can be obtained by solving the above model repeatedly, which forms the efficient frontier (EF). For each point on EF, there should be no portfolio with a higher expected return at the same risk level, and no portfolio with a lower risk at the same level of return.

\subsection{Additional Practical Constraints}

Several extensions have been proposed to enrich the MV model with real world constraints in the literature. In this paper, we investigate the following mostly studied additional practical constraints based on the basic MV model.

Cardinality constraint The cardinality constraint restricts the number of $K$ assets in a portfolio. A binary variable $z_{i}$ is introduced to denote whether an asset is selected or not. This constraint is relaxed to i.e. $\sum_{i=1}^{N} z_{i} \leq K$ in some work in the literature.

$$
\begin{aligned}
& \sum_{i=1}^{N} z_{i}=K \\
& z_{i} \in\{0,1\}, i=1, \ldots, N
\end{aligned}
$$

Quantity constraint The quantity constraint specifies the lower $(\varepsilon)$ and upper $(\delta)$ bounds allowed for the allocated proportions to each asset in a portfolio.

$$
\varepsilon z_{i} \leqslant x_{i} \leqslant \delta z_{i}, i=1, \ldots, N
$$

Pre-assignment constraint Pre-assignment constraint pre-selects investor's preferred assets in the portfolio. It was firstly discussed in (Chang et al., 2000) and firstly applied in (Di Gaspero et al., 2011). A binary variable $s_{i}$ is introduced to denote if asset $i$ belongs to the pre-assigned set $P, 0$ otherwise.

$$
\begin{aligned}
& s_{i}=1, i \in P \\
& z_{i} \geqslant s_{i}, i=1, \ldots, N
\end{aligned}
$$

Round lot constraint Round lot constraint defines that the investment of any asset in the portfolio should be an exact multiple units of a minimum lot. An integer variable $y_{i}$ and minimum lot $l_{i}$ for each asset are introduced. The round lot constraint might cause the budget constraint (3) not strictly satisfied, as the capital cannot always be divided as an exact multiple of trading lot for all the assets.

$$
x_{i}=y_{i} * l_{i}, i=1, \ldots, N
$$

Class constraint Introduced by (Chang et al., 2000), class constraint is used to limit the total proportion invested in those assets with common characteristics, leading to a more diversified and safe portfolio. Classes of assets are considered to be mutually exclusive, i.e. $C_{i} \cap C_{j}=\varnothing$ for all assets $i \neq j$. In this study we require at least one asset from each of the M classes to be selected, thus $K \geq M$. Here the upper bound is set as 1 , and the lower bound of each class is $L_{m}>0$ for every class $C_{m}, m=1, \ldots, M$. Then the bound constraint is formulated as follows:

$$
L_{m} \leqslant \sum_{i \in C_{m}} x_{i} \leqslant 1, m=1, \ldots M
$$

\section{STUDIES OF VARIOUS EXTENDED MV MODELS}

The basic MV model is a quadratic programming problem and can be solved efficiently by some specialized exact methods such as simplex method and 
branch and bound methods. These techniques can also handle arbitrary linear constraints, like quantity constraint, see (Borchers and Mitchell, 1997). Nevertheless the problem becomes increasingly much more complex when the number of assets increases and with additional constraints. For instance, with the cardinality constraint, the problem turns into a mixed integer nonlinear programming and NP-hard (Bienstock, 1995). (Bienstock, 1995) presented a branch and cut algorithm for the cardinality constrained PO problem of up to 3897 assets with various cardinality values. At the time of publication results shown that this type of problem with larger size may be impossible to solve to a proved optimality within a reasonable time. Some works require strictly $K$ assets to be included in a portfolio (/Chang et al., 2000; Fernandez and Gomez, 2007; Xu et al., 2010, Woodside-Oriakhi et al., 2011; Jin et al., 2014)) while some others use relaxed version ((Schaerf, 2002, Ruiz-Torrubiano and Suarez, 2010)).

There are not many experimental studies on the pre-assignment constraint so far, except some informal discussion (Di Tollo and Roli, 2008). (Di Gaspero et al., 2011) examined the impact of the preassignment constraint and reported that pre-assigning one asset tended to worsen the performance except when the asset is in the optimal solution for all the expected return levels.

Recently, some scholars included the round-lot constraint into PO problems, which makes it more difficult to find a feasible solution. Some of these were measured in units of money ((Speranza, 1996, Mansini and Speranza, 1999, Kellerer et al., 2000. Lin and Liu, 2008, Bonami and Lejeune, 2009, Golmakani and Fazel, 2011)), while others imposed that the continuous weight variables should be an integer multiple of a given fraction ((Jobst et al., 2001; Streichert et al., 2004; Skolpadungket et al., 2007)). Some of them included transaction cost at the same time. (Mansini and Speranza, 1999) showed that a PO problem with minimum lot and without any fixed transaction cost is NP-complete.

Class constraint was first introduced by (Chang et al., 2000), also mentioned in (Ruiz-Torrubiano and Suarez, 2010), and applied in ((Anagnostopoulos and Mamanis, 2010, Anagnostopoulos and Mamanis, 2011a Anagnostopoulos and Mamanis, 2011b)). Another form of this constraint is splitting the universe of assets into subsets with similar features. Optimisation is performed on the best representative of each class (Vijayalakshmi Pai and Michel, 2009).

Most of the research in the literature adopt two constraints in the problem formulation. In particular, cardinality and quantity constraints were stud- ied $44.93 \%$ and $30.43 \%$, respectively, in the portfolio management models (Metaxiotis and Liagkouras, 2012).

\section{ANALYSIS ON DIFFERENT CONSTRAINTS IN THE MV MODEL}

As most of the applications in the literature dealt with quantity and cardinality constrained PO problems, in this section we first discuss the effect of pre-assignment, round-lot and class constraint based on the quantity and cardinality constrained MV model in terms of solution quality and computational cost. Then we present experimental framework using CPLEX 12.6 to identify solutions for the most commonly applied constrained MV models in the literature, some models with different settings in constraints.

Five widely tested benchmark datasets in the ORlibrary (Beasley, 1990) (http://people.brunel. ac.uk/ mastjjb/jeb/info.html) are chosen in our experiments. They were extracted from the wellknown indices, the Hong Kong HangSeng, the German DAX100, the UK FTSE100, the US S\&P100 and the Japan Nikkei, with dimension $N=31,85,89,98$ and 225 (N31-N225), respectively. The portfolios obtained under different constraints and settings form constrained efficient frontiers (CEF). The unconstrained efficient frontier (UEF), for the unconstrained PO problem from the OR-library, is used as the upper bounds for CEF. For each dataset, 50 points across the UEF are chosen. For each point on the $\mathrm{CEF}$, the solver is run to minimise risk $(\mathrm{QP})$ given a respected return. The stopping time limit is set to 3600 seconds.

Several performance measures are adopted, for which smaller values denote better solutions. The Average Percentage Error (APE) (Di Gaspero et al., 2011) measures the relative distance between the obtained CEF from the UEF with the same expected return, calculated using Formula (11), where $x^{*}$ and $x$ denote the portfolios on the CEF and UEF, respectively, $f_{i}^{r}$ denotes the value of the obtained risk, and $p$ is the number of portfolios on the frontier.

$$
A P E=\frac{1}{p} * \sum_{i=1}^{p} \frac{f_{i}^{r}\left(x^{*}\right)-f_{i}^{r}(x)}{f_{i}^{r}(x)}
$$

Generational distance (GD) (Cura, 2009) refers to the average minimum distance of each portfolio on the CEF from the UEF, calculated using Formula (12) where $d_{x^{*} x}$ is the Euclidean distance. $f_{v}$ and $f_{r}$ are the 
risk (Eq.(1)) and return (Eq.(2)) of the portfolio. It measures how close the obtained CEF is to the UEF.

$$
\begin{aligned}
G D & =\frac{1}{p} * \sum_{x^{*} \in X_{C E F}} \min \left\{d_{x^{*} x} \mid x \in X_{U E F}\right\} \\
d_{x^{*} x} & =\sqrt{\left(f_{r}\left(x^{*}\right)-f_{r}(x)\right)^{2}+\left(f_{v}\left(x^{*}\right)-f_{v}(x)\right)^{2}}
\end{aligned}
$$

Inverted generational distance(IGD) is a variant of GD. It uses UEF as a reference to calculate the average minimum distance of its each portfolio to the CEF, calculated using Formula (14). This measure mainly shows the overall quality of the obtained solution set (i.e. its diversity and convergence to the UEF).

$$
I G D=\frac{1}{p} * \sum_{x^{*} \in X_{U E F}} \min \left\{d_{x^{*} x} \mid x \in X_{C E F}\right\}
$$

Algorithm Effort (AE) (Chen et al., 2012), $A E=$ $\frac{\text { Time }}{p}$, measures the ratio of the total run time to the number of feasible portfolios obtained on the CEF. MAX and MIN denote the maximum or minimum time spent for one portfolio. Optimal Rate is used to denote the rate of optimal solutions obtained out of all the points on the $\mathrm{CEF}$.

The experiments are coded in $\mathrm{C}++$ in Microsoft Visual Studio 2012, and run on a PC with Windows 7 Operating System (64-bit), 6GB of RAM, and an Intel Core i7 CPU (960@3.2GHz).

\subsection{Pre-assignment, Round-lot and Class constraints}

We first test all the extended constraints on the MV model. The lower and upper bounds of the weights are set as $\varepsilon=0.01, \delta=1$, and cardinality $K=10$. These are widely used in the literature. Preliminary computational results indicate that the pre-assigned asset(s) is not a main performance discriminator of the basic MV model, thus is randomly set as $P=$ $\{30\}$. The class constraints is set to: randomly define two classes with size of $20 \%$ proportionately to problem dimension $(N)$. These constraints are set as those in (Lwin et al., 2014). Initial experiment results showed that cardinality constraint contributes the most to the problem difficulty.

We then assess the effect on the computational cost from pre-assignment (C3), round-lot (C4) and class (C5) constraints based on the MV model with cardinality $(\mathrm{C} 1)$ and quantity $(\mathrm{C} 2)$ constraints, i.e. $\varepsilon=0.01, K=10$.

\subsubsection{Pre-assignment Constraint(C3)}

To evaluate the impact of pre-assignment constraint on the computational cost, for each benchmark in- stance, we fix in turn one of the assets as pre-assigned (imposing $\mathrm{C} 1$ and $\mathrm{C} 2$ with the settings as mentioned above). For each instance we then obtain a group of $N$ $\mathrm{CEFs}$, one for each pre-assigned asset. Intuitively, the choice of the pre-assigned assets determines the solution quality measured in APE. For example, if the pre-assigned asset does not belong to any optimal solution for most of the values of the required return $R_{\text {exp }}$ on UEF, the pre-assignment constraint generally deteriorates the solution quality. Moreover, the magnitude of this worsening may depend on the features of the asset, e.g. return rate, standard deviation(sd), and the ratio of return/sd, etc.

As an example, in Figure 1, for instance N98, the average time spent for computing the CEF for each pre-assigned asset is plotted. It can be seen that the time used varies with obvious difference among different assets, ranging from around 20s to 400s. Figure 2 reports the frequency of each asset appearing in the optimal portfolios on the UEF. For instance, asset 1 which leads to the maximum time used (400s) never appears in the optimal solution in the unconstrained problem. Moreover, the rankings of its return rate, $\mathrm{sd}$, and the ratio of return/sd are quite low, ranked as 61,64 , and 71 , respectively. The assets ranked top in return rate, sd and ratio (namely assets 82, 62, and 89 ) appear to lead to around only 50s, which is at the lower level of time used in Figure 1 Moreover, asset 89 appears 36 times out of 50 in the optimal portfolios on the UEF shown in Figure 2. These imply that there is some correlation between the feature of the pre-assigned asset and the solution construction.

Intuitively, if we pre-assign more assets in advance, the problem becomes smaller with only $(K-$ pre_assigned) number of assets. We conduct an another experiment on two groups of pre-assigned assets on instance N98, each with the top five and bottom five shown in Figure 2. As expected in Table 1, the computational time with more pre-assigned assets is much lower than that with fewer assets. Pre-assigning the assets which intend to appear in the optimal portfolio on UEF can reduce the computational cost and lead to better solution quality.

Table 1: Results of pre-assigned assets with different features

\begin{tabular}{lcccc}
\hline Pre-assigned & APE & MAX & AE & Optimal Rate \\
\hline Top 5 & 0.100718 & 19.005 & 4.609 & 0.98 \\
Bottom 5 & 0.234882 & 122.136 & 22.716 & 0.96 \\
\hline
\end{tabular}

\subsubsection{Round-lot Constraint(C4)}

We test on all the instances with three different lot unit sizes and compare the results against the model 


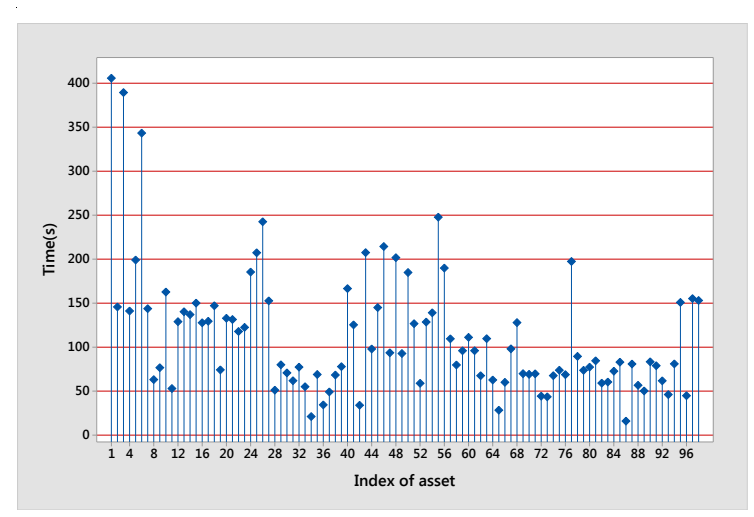

Figure 1: Average time used for N98, with each preassigned asset

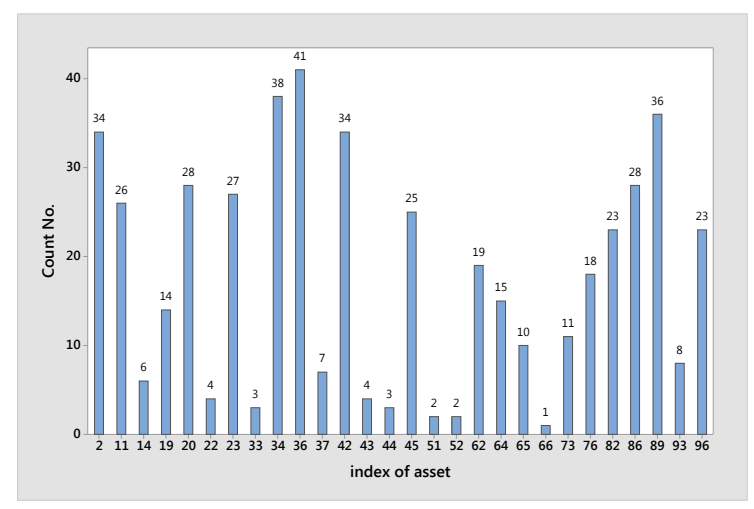

Figure 2: Frequency of each in the optimal solution on UEF in $\mathrm{N} 98, \mathrm{y}$-axix is the occurrence number

without round-lot constraint. As seen in Table 2 , there are no significant differences in terms of the computational time and optimal rate. The chosen lot unit size has a slight effect on the optimal rate.

Table 2: Results on the $\mathrm{C} 4$ constrained problem based on $\mathrm{C} 1$ and $\mathrm{C} 2$ constrained $\mathrm{MV}$ model

\begin{tabular}{cccccc}
\hline Dataset & Lot unit & $\mathrm{APE}$ & Max Time & $\mathrm{AE}$ & Optimal Rate \\
\hline & $\mathrm{NA}$ & 0.02107 & 0.983 & 0.138 & 0.92 \\
& 0.005 & 0.02157 & 0.719 & 0.269 & 0.92 \\
& 0.008 & 0.03321 & 9.612 & 2.675 & 0.88 \\
$\mathrm{~N} 31$ & 0.01 & 0.02194 & 0.577 & 0.108 & 0.92 \\
\hline & $\mathrm{NA}$ & 0.07539 & 61.463 & 11.500 & 0.94 \\
& 0.005 & 0.07686 & 49.954 & 5.550 & 0.94 \\
$\mathrm{~N} 85$ & 0.008 & 0.07686 & 49.954 & 5.550 & 0.94 \\
& 0.01 & 0.07838 & 44.33 & 5.03887 & 0.94 \\
\hline & $\mathrm{NA}$ & 0.02569 & 902.803 & 101.885 & 0.94 \\
& 0.005 & 0.02625 & 1586.61 & 97.140 & 0.94 \\
$\mathrm{~N} 89$ & 0.008 & 0.02936 & 400.068 & 39.773 & 0.92 \\
& 0.01 & 0.02681 & 1177.83 & 91.809 & 0.94 \\
\hline & $\mathrm{NA}$ & 0.06181 & 3600.59 & 1055.72 & 0.7 \\
& 0.005 & 0.06265 & 3600.47 & 1042.23 & 0.72 \\
$\mathrm{~N} 98$ & 0.008 & 0.06749 & 3600.64 & 979.171 & 0.76 \\
& 0.01 & 0.06345 & 3600.4 & 1037.42 & 0.72 \\
\hline & $\mathrm{NA}$ & 0.01034 & 16.333 & 7.229 & 0.98 \\
& 0.005 & 0.01107 & 12.592 & 5.284 & 0.98 \\
$\mathrm{~N} 225$ & 0.008 & 0.01129 & 23.865 & 8.139 & 0.96 \\
& 0.01 & 0.01138 & 9.441 & 4.146 & 0.98 \\
\hline & & & & &
\end{tabular}

\subsubsection{Class Constraint(C5)}

The class constraint is tested with different settings on the class defined and the lower bound. From the theoretical perspective, class constraint is linear thus does not increase the problem difficulty. The number of classes should also reduce the problem difficulty as the original problem is partitioned into a set of subproblems with lower dimension. Table 3 shows the results obtained on instance N98 with different class settings based on the $\mathrm{C} 1$ and $\mathrm{C} 2$ constrained models. The class classification here is randomly assigned.

From Table 3 it can be seen that the higher lower bound 0.1 could reduce the optimal rate compared to 0.05 . As expected, the setting with less classes requires much more computational effort than those with more classes. Intuitively expected, in terms of computational cost, the class constraint does not generate much difficulty on the cardinality constrained model, while it can affect the rate of optimality.

Table 3: Results with different class settings based on the $\mathrm{C} 1$ and $\mathrm{C} 2$ constrained MV Model

\begin{tabular}{lcccc}
\hline Class setting & APE & MAX & AE & Optimal Rate \\
\hline NA & 0.061814 & 3600.59 & 1055.72 & 0.7 \\
size $=6$, LC=0.05 & 0.163718 & 519.235 & 101.793 & 0.84 \\
size $=6$, LC=0.1 & 0.246108 & 999.286 & 156.248 & 0.68 \\
size $=2$, LC=0.1 & 0.096405 & 3601.05 & 1123.34 & 0.62 \\
size $=2$, LC $=0.05$ & 0.079334 & 3599.82 & 433.568 & 0.9 \\
\hline
\end{tabular}

\subsection{Performance on the Complete Model}

Table 4 reports our results under different constraint settings (Columns C1-C5) in terms of optimality, solution quality (GD, IGD, APE), and computational cost (MAX, MIN, AE) for the constrained models. This feature is varied by the researchers. In the last two models, for each instance, the asset which took the longest time in Figure 1 is pre-assigned. Two classes are defined in the class constraint with the lower bound set as 0.05 . The results illustrate that, the last two models spent much less computational cost compared to the $\mathrm{C} 1$ and $\mathrm{C} 2$ constrained models. In other words, combination of all these constraints seems to have neutralising effect.

\subsection{Performance on Existing MV Models in the Literature}

In addition, to provide the optimal or lower bound for various subsets of the constrained MV models in the literature, the most commonly applied settings in different constraints in relevant works are tested. Due to 


\begin{tabular}{|c|c|c|c|c|c|c|}
\hline 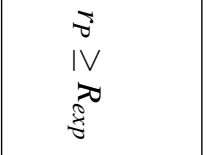 & 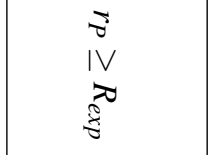 & $\begin{array}{l}\overrightarrow{0} \\
11 \\
8 \\
8\end{array}$ & $\begin{array}{l}\overrightarrow{0} \\
\text { IV } \\
\substack{0 \\
0}\end{array}$ & \begin{tabular}{l}
$\overrightarrow{0}$ \\
$\|$ \\
\multirow{8}{0}{}
\end{tabular} & $\begin{array}{l}\text { IV } \\
\text { IV } \\
\substack{0 \\
0}\end{array}$ & 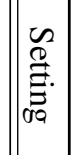 \\
\hline $\begin{array}{l}\pi \\
\| \\
0\end{array}$ & $\begin{array}{l}\pi \\
\| \\
0\end{array}$ & $\begin{array}{l}\bar{\lambda} \\
1 \wedge \\
0\end{array}$ & $\begin{array}{l}\lambda \\
11 \\
0\end{array}$ & $\begin{array}{l}\pi \\
\ddot{0}\end{array}$ & $\begin{array}{l}\pi \\
\| \\
0\end{array}$ & $\Omega$ \\
\hline $\begin{array}{l}0 \\
11 \\
0 \\
0\end{array}$ & $\begin{array}{l}\infty \\
11 \\
0 \\
0\end{array}$ & $\begin{array}{l}\infty \\
11 \\
0 \\
0\end{array}$ & $\begin{array}{l}\infty \\
11 \\
0 \\
0\end{array}$ & $\begin{array}{l}\infty \\
\| \\
0 \\
0\end{array}$ & $\begin{array}{l}\infty \\
\| \\
0 \\
0\end{array}$ & $\mathcal{N}$ \\
\hline 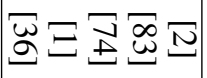 & 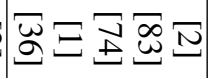 & & & & & $\mathcal{\omega}$ \\
\hline$\stackrel{8}{8}$ & $\stackrel{8}{8}$ & & & & & $\AA$ \\
\hline 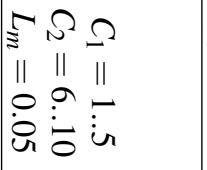 & 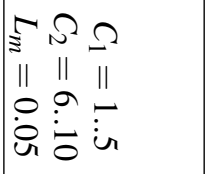 & & & & & O \\
\hline 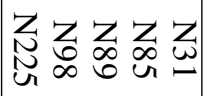 & 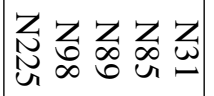 & 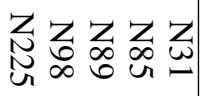 & 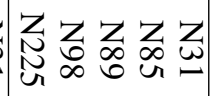 & 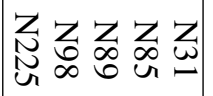 & 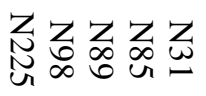 & 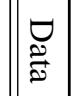 \\
\hline 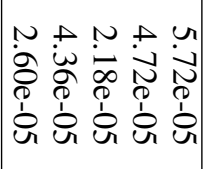 & 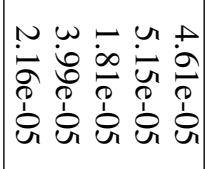 & 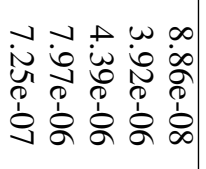 & 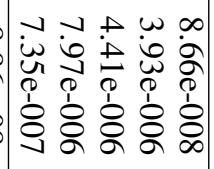 & 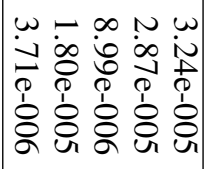 & 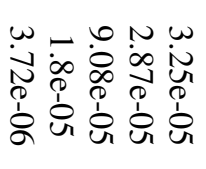 & $\Omega$ \\
\hline 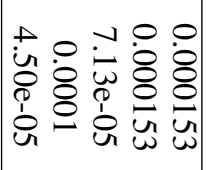 & 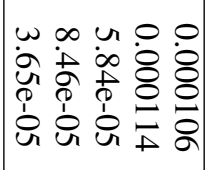 & 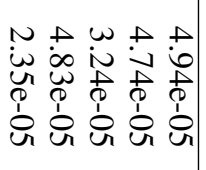 & 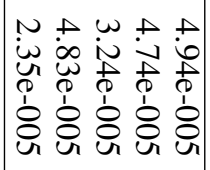 & 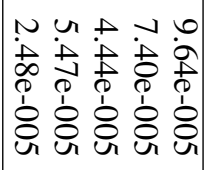 & 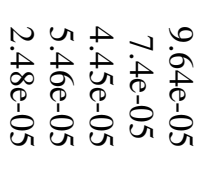 & $\bar{\Omega}$ \\
\hline 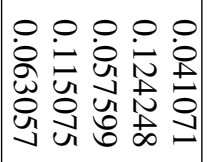 & 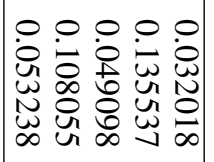 & 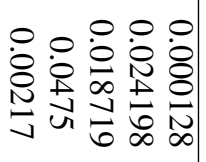 & 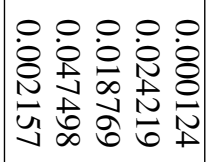 & 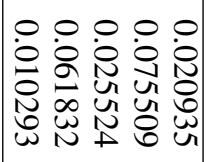 & 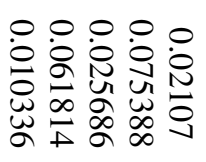 & 悬 \\
\hline 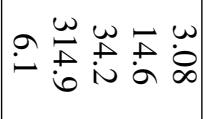 & 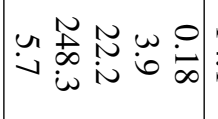 & 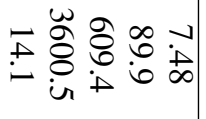 & 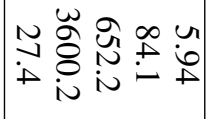 & 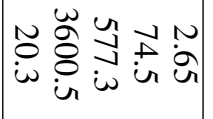 & 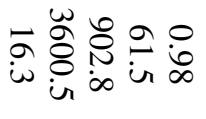 & 3 \\
\hline 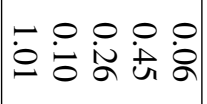 & 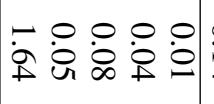 & 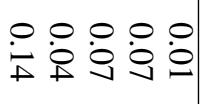 & 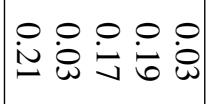 & 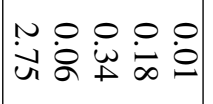 & $\begin{array}{l}N \\
\text { üs }\end{array}$ & 争 \\
\hline 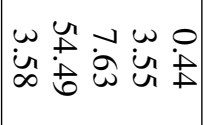 & 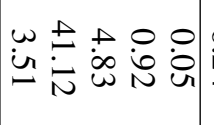 & 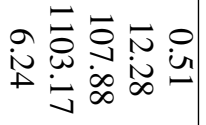 & 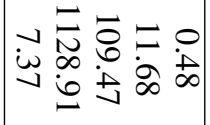 & 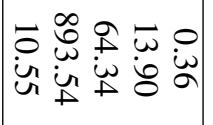 & 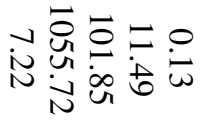 & 尚 \\
\hline 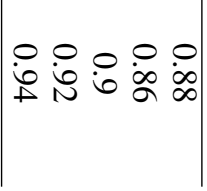 & 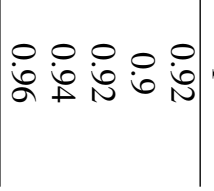 & $-\stackrel{\ominus}{-}$ & $-\stackrel{\circ}{-}$ & 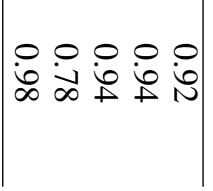 & 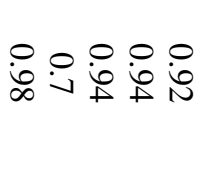 & 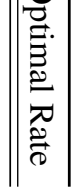 \\
\hline
\end{tabular}


Table 5: Results for various models in literature

\begin{tabular}{l|l|l|l|l|l|l|}
\hline Models in the literature & Data & GD & IGD & APE & AE & Optimal Rate \\
\hline Maringer and Kellerer, 2003 & N31 & $5.82 \mathrm{E}-08$ & $4.94 \mathrm{E}-05$ & $8.20 \mathrm{E}-05$ & 0.01176 & 1 \\
& N85 & $3.91 \mathrm{E}-06$ & $4.74 \mathrm{E}-05$ & 0.024185 & 0.66952 & 1 \\
& N89 & $4.40 \mathrm{E}-06$ & $3.24 \mathrm{E}-05$ & 0.018769 & 1.95914 & 1 \\
& N98 & $7.96 \mathrm{E}-06$ & $4.83 \mathrm{E}-05$ & 0.047486 & 10.6813 & 1 \\
& N225 & $6.76 \mathrm{E}-07$ & $2.35 \mathrm{E}-05$ & 0.002015 & 1.2937 & 1 \\
\hline Chang et al., 2000 & N31 & $3.25 \mathrm{E}-05$ & $9.64 \mathrm{E}-05$ & 0.02107 & 0.137674 & 0.92 \\
\hline Fernandez and Gomez, 2007 & N85 & $2.87 \mathrm{E}-05$ & $7.40 \mathrm{E}-05$ & 0.075388 & 11.4999 & 0.94 \\
\hline Xu et al., 2010 & N89 & $9.08 \mathrm{E}-06$ & $4.45 \mathrm{E}-05$ & 0.025686 & 101.885 & 0.94 \\
$K=10$ & N98 & $1.80 \mathrm{E}-05$ & $5.46 \mathrm{E}-05$ & 0.061814 & 1055.72 & 0.7 \\
$\varepsilon=0.01$ & N225 & $3.72 \mathrm{E}-06$ & $2.48 \mathrm{E}-05$ & 0.010336 & 7.22906 & 0.98 \\
\hline Ruiz-Torrubiano and Suarez, 2010 & N31 & $8.86 \mathrm{E}-08$ & $4.94 \mathrm{E}-05$ & 0.000128 & 0.51264 & 1 \\
\hline Schaert, 2002 & N85 & $3.92 \mathrm{E}-06$ & $4.74 \mathrm{E}-05$ & 0.024198 & 12.2765 & 1 \\
$K<=10$ & N89 & $4.39 \mathrm{E}-06$ & $3.24 \mathrm{E}-05$ & 0.018719 & 107.883 & 1 \\
$\varepsilon=0.01$ & N98 & $7.97 \mathrm{E}-06$ & $4.83 \mathrm{E}-05$ & 0.0475 & 1103.17 & 0.7 \\
& N225 & $7.25 \mathrm{E}-07$ & $2.35 \mathrm{E}-05$ & 0.00217 & 6.2358 & 1 \\
\hline Lwin et al., 2014 & N31 & $5.38 \mathrm{E}-05$ & 0.00015 & 0.036569 & 1.90675 & 0.88 \\
$K=10$ & N85 & $4.14 \mathrm{E}-05$ & 0.000108 & 0.104911 & 16.5399 & 0.9 \\
$\varepsilon=0.01$ & N89 & $2.22 \mathrm{E}-05$ & $5.93 \mathrm{E}-05$ & 0.054954 & 12.4044 & 0.92 \\
& N98 & $2.68 \mathrm{E}-05$ & $6.68 \mathrm{E}-05$ & 0.084467 & 107.943 & 0.96 \\
& N225 & $1.74 \mathrm{E}-05$ & $3.86 \mathrm{E}-05$ & 0.04161 & 7.39513 & 0.94 \\
\hline Woodside-Oriakhi et al., 2011 & N31 & $2.66 \mathrm{E}-05$ & 0.000159 & NA & 0.0766 & 1 \\
$K=10$ & N85 & $1.08 \mathrm{E}-05$ & 0.000128 & NA & 9.91606 & 1 \\
$\varepsilon=0.01$ & N89 & $6.81 \mathrm{E}-06$ & $9.35 \mathrm{E}-05$ & NA & 94.7458 & 1 \\
$R_{\text {exp }}$ in range:[0.9R $\left.R_{\text {exp }}, 1.1 R_{\text {exp }}\right]$ & N98 & $1.46 \mathrm{E}-05$ & 0.00014 & NA & 1105.7 & 0.72 \\
& N225 & $2.06 \mathrm{E}-06$ & $5.27 \mathrm{E}-05$ & NA & 6.54972 & 1 \\
\hline
\end{tabular}

the space limited, a sketch of the performance of the representative models with different combinations of constraints is shown in Table 5. More detailed data and updated models are published in http://www. cs.nott.ac.uk/ pszrq/benchmarks.htm.

\section{CONCLUSION AND FUTURE WORK}

This paper studies the constrained Markowitz MV model for the portfolio optimisation problem, where quantity, cardinality, pre-assignment, round-lot and class constraints are considered. We first discuss the effect of the pre-assignment, round-lot and class constraints based on the cardinality and quantity constrained models in terms of computational cost. Then we conduct experiments using CPLEX to obtain optimal or feasible solutions within a limited computational cost for various models with different constraint settings. The results for the thoroughly studied constrained models are presented.

According to the results, the pre-assignment, round-lot and class constraints do not make a big difference to the cost of solving the quantity and cardinality constrained problem. As the mostly considered constraint in the current literature, the cardinality constraint is proved to contribute to mainly the problem difficulty. In addition, the more specific settings imposed on these constraints, the easier the problem is. The complete constrained MV model with all the constraints can neutralize the effect of cardinality constraint, and the optimal solutions can be much easier to obtain.
There are some exceptional points on instance N98, which still requires a huge amount of computational effort for the cardinality constrained models. Nevertheless, due to the fast development of commercial solvers, most of the currently studied constrained MV models can be efficiently solved to obtain most of the optimal solutions within a limited time.

The above results motivate our future research to more challenging PO problem. In the future, we plan to further study the constrained PO problem based on other risk measures such as $\mathrm{VaR}$ and $\mathrm{CVaR}$ which are favoured by investors in reality. It is also interesting to consider other constraints such as transaction cost which occurs for problem with more than one investment period. We also aim to analyse the performance on difficult real instances of larger problem size.

\section{REFERENCES}

Anagnostopoulos, K. and Mamanis, G. (2010). A portfolio optimization model with three objectives and discrete variables. Computers \& Operations Research, 37(7):1285 - 1297. Algorithmic and Computational Methods in Retrial Queues.

Anagnostopoulos, K. and Mamanis, G. (2011a). The meanvariance cardinality constrained portfolio optimization problem: An experimental evaluation of five multiobjective evolutionary algorithms. Expert Systems with Applications, 38(11):14208 - 14217.

Anagnostopoulos, K. P. and Mamanis, G. (2011b). Multiobjective evolutionary algorithms for complex portfolio optimization problems. Computational Management Science, 8(3):259-279.

Beasley, J. E. (1990). Or-library: Distributing test prob- 
lems by electronic mail. Journal of the Operational Research Society, 41:1069-1072.

Bienstock, D. (1995). Computational study of a family of mixed-integer quadratic programming problems. In Integer Programming and Combinatorial Optimization, volume 920 of Lecture Notes in Computer Science, pages 80-94. Springer Berlin Heidelberg.

Bonami, P. and Lejeune, M. A. (2009). An exact solution approach for portfolio optimization problems under stochastic and integer constraints. Operations Research, 57(3):650-670.

Borchers, B. and Mitchell, J. E. (1997). A computational comparison of branch and bound and outer approximation algorithms for 01 mixed integer nonlinear programs. Computers \& Operations Research, 24(8):699 -701 .

Chang, T.-J., Meade, N., Beasley, J., and Sharaiha, Y. (2000). Heuristics for cardinality constrained portfolio optimisation. Computers \& Operations Research, 27(13): $1271-1302$.

Chen, A., Liang, Y.-C., and Liu, C.-C. (2012). An artificial bee colony algorithm for the cardinalityconstrained portfolio optimization problems. In 2012 IEEE Congress on Evolutionary Computation (CEC), pages $1-8$.

Cura, T. (2009). Particle swarm optimization approach to portfolio optimization. Nonlinear Analysis: Real World Applications, 10(4):2396 - 2406.

Di Gaspero, L., Di Tollo, G., Roli, A., and Schaerf, A. (2011). Hybrid metaheuristics for constrained portfolio selection problems. Quantitative Finance, 11(10):1473-1487.

Di Tollo, G. and Roli, A. (2008). Metaheuristics for the portfolio selection problem. International Journal of Operationas Research, 5(1):13-35.

Fernandez, A. and Gomez, S. (2007). Portfolio selection using neural networks. Computers \& Operations Research, 34(4): 1177 - 1191.

Golmakani, H. R. and Fazel, M. (2011). Constrained portfolio selection using particle swarm optimization. Expert Systems with Applications, 38(7):8327 - 8335.

Jansen, R. and van Dijk, R. (2002). Optimal benchmark tracking with small portfolios. The Journal of Portfolio Management, 28(2):33-39.

Jin, Y., Qu, R., and Atkin, J. (2014). A population-based incremental learning method for constrained portfolio optimisation. In Symbolic and Numeric Algorithms for Scientific Computing (SYNASC), 2014 16th International Symposium on, pages 212-219.

Jobst, N., Horniman, M., Lucas, C., and Mitra, G. (2001). Computational aspects of alternative portfolio selection models in the presence of discrete asset choice constraints. Quantitative Finance, 1(5):489-501.

Kellerer, H., Mansini, R., and Speranza, M. (2000). Selecting portfolios with fixed costs and minimum transaction lots. Annals of Operations Research, 99(14):287-304.

Lin, C.-C. and Liu, Y.-T. (2008). Genetic algorithms for portfolio selection problems with minimum transac- tion lots. European Journal of Operational Research, 185(1):393 - 404.

Lwin, K., Qu, R., and Kendall, G. (2014). A learningguided multi-objective evolutionary algorithm for constrained portfolio optimization. Applied Soft Computing, 24:757 - 772 .

Mansini, R. and Speranza, M. G. (1999). Heuristic algorithms for the portfolio selection problem with minimum transaction lots. European Journal of Operational Research, 114(2):219 - 233.

Maringer, D. (2008). Heuristic optimization for portfolio management [application notes]. Computational Intelligence Magazine, IEEE, 3(4):31 -34.

Maringer, D. and Kellerer, H. (2003). Optimization of cardinality constrained portfolios with a hybrid local search algorithm. OR Spectrum, 25(4):481-495.

Markowitz, H. (1952). Portfolio selection. The Journal of Finance, 7(1):pp. 77-91.

Metaxiotis, K. and Liagkouras, K. (2012). Multiobjective evolutionary algorithms for portfolio management: A comprehensive literature review. Expert Systems with Applications, 39(14):11685 - 11698.

Ruiz-Torrubiano, R. and Suarez, A. (2010). Hybrid approaches and dimensionality reduction for portfolio selection with cardinality constraints. Computational Intelligence Magazine, IEEE, 5(2):92-107.

Schaerf, A. (2002). Local search techniques for constrained portfolio selection problems. Computational Economics, 20:177-190.

Skolpadungket, P., Dahal, K., and Harnpornchai, N. (2007). Portfolio optimization using multi-objective genetic algorithms. In Evolutionary Computation, 2007. CEC 2007. IEEE Congress on, pages 516-523.

Speranza, M. G. (1996). A heuristic algorithm for a portfolio optimization model applied to the milan stock market. Computers \& Operations Research, 23(5):433 441.

Streichert, F., Ulmer, H., and Zell, A. (2004). Evaluating a hybrid encoding and three crossover operators on the constrained portfolio selection problem. In Evolutionary Computation, 2004. CEC2004. Congress on, volume 1, pages 932-939.

Vijayalakshmi Pai, G. and Michel, T. (2009). Evolutionary optimization of constrained $\mathrm{k}$-means clustered assets for diversification in small portfolios. Evolutionary Computation, IEEE Transactions on, 13(5):1030 1053.

Woodside-Oriakhi, M., Lucas, C., and Beasley, J. (2011). Heuristic algorithms for the cardinality constrained efficient frontier. European Journal of Operational Research, 213(3):538 - 550.

Xu, R.-t., Zhang, J., Liu, O., and Huang, R.-Z. (2010). An estimation of distribution algorithm based portfolio selection approach. In Technologies and Applications of Artificial Intelligence (TAAI), 2010 International Conference on, pages 305-313. 